\title{
Risk Stratification in Dialysis Patients: Coronary Artery Calcification Score Combined with High Sensitive C-Reactive Protein and Framingham Score for Cardiovascular Risk Prediction in Asymptomatic Subjects
}

Katarzyna Janda ${ }^{1}$, Marcin Krzanowski ${ }^{1}$, Paulina Dumnicka ${ }^{2}$, Maria Kapusta ${ }^{3}$, Piotr Klimeczek ${ }^{4}$, Eve Chowaniec ${ }^{1}$, Przemysław Miarka ${ }^{1}$, Mieczysław Pasowicz ${ }^{4}$ and Władysław Sułowicz

${ }^{1}$ Department of Nephrology, Jagiellonian University, Medical College, Cracow, Poland

${ }^{2}$ Department of Medical Diagnostics, Jagiellonian University, Medical College, Cracow, Poland

${ }^{3}$ Chair of Clinical Biochemistry, Jagiellonian University, Medical College, Cracow, Poland

${ }^{4}$ Center for Diagnosis, Prevention and Telemedicine, John Paul II Hospital, Cracow, Poland

\begin{abstract}
Introduction: Vascular calcification independently predicts cardiovascular disease, the major cause of death in Chronic Kidney Disease (CKD) patients. Coronary Artery Calcium Score (CACS) is a marker for atherosclerotic plaque burden, vascular calcification and has been shown to be a predictor of incidence of myocardial infarction and death from Cardiovascular (CV) disease.
\end{abstract}

Objectives: The aim of the study was to evaluate factors influencing CV mortality in a group of Peritoneal Dialysis $(P D)$ patients during a six year observation period.

Patients and methods: The study included 53 patients with no symptoms of CV disease (25 women, 28 men; mean age of $52 \pm 12$ years) treated with PD for a median period of 24 months. Baseline Framingham Risk Score (FRS) was assessed and CACS was measured using Multi-Row Spiral Computed Tomography (MSCT). Laboratory measurements included high sensitive C-reactive protein (hsCRP), osteoprotegerin (OPG), fibroblast growth factor 23 (FGF23), osteopontin (OPN), osteocalcin (OC), intact parathyroid hormone (iPTH), total calcium (Ca) and phosphates $(\mathrm{Pi})$. The data concerning mortality was collected over a 6 year period.

Results: During the six year observation period, 24 (45\%) patients died, including 19 due to CV causes. Median overall survival was 72 months (lower quartile, 17 months). CACS was a significant predictor of all-cause and CV mortality both in simple analysis ( $H R=1.03$ per 100 Agatston units, $p=0.02$ and $H R=1.05, p=0.003$ ), as well as in a multiple model adjusted for age of patients, dialysis duration, weekly creatinine clearance, $\mathrm{Ca} \times \mathrm{Pi}$, iPTH, OPG, hsCRP and FRS ( $H R=1.04, p=0.02$ and $H R=1.05, p=0.01)$. The value of 800 Agatston units significantly differentiated the group into those with higher and lower risk for CV death $(p=0.04)$. Age and FGF23 concentration were independent predictors of CACS. Also, hsCRP and FRS significantly predicted all-cause and CV mortality in simple Cox regression ( $H R=1.04, p=0.002$ and $H R=1.04, p=0.003 ; H R=1.14, p=0.047$ and $H R=1.23, p=0.01$ ) as well as in a multiple model $(H R=1.05, p=0.002$ and $H R=1.05, p=0.01 ; H R=1.23, p=0.01$ and $H R=1.33, p=0.004)$. Adding CACS to FRS and hsCRP significantly improved the prediction of cardiovascular mortality $(p=0.02)$.

Conclusions: Coronary calcium imaging is a non-invasive method of $\mathrm{CV}$ risk stratification that can accurately identify high-risk asymptomatic dialysis patients at the start of dialysis. The assessment of CACS together with inflammatory markers and conventional CV risk factors (FRS) may contribute to early diagnosis, prevention and reduction of deaths from CV disease in dialysis patients. Among the markers of bone disease, FGF-23 (a regulator of phosphorus metabolism) may be an early predictor of vascular calcification among dialysis patients.

Keywords: Calcium scoring; Cardiovascular mortality; Fibroblast growth factor 23; Framingham risk score; Osteoprotegerin; Peritoneal dialysis

\section{Introduction}

Vascular calcification independently predicts cardiovascular (CV) disease, the major cause of death In Chronic Kidney Disease (CKD) patients. Coronary Artery Calcium Score (CACS) is a marker for atherosclerotic plaque burden and has been shown to predict the incidence of myocardial infarction and death from CV disease. CKD leads to increased calcium score secondary to accelerated progression of atherosclerosis, as well as changes in calcium homeostasis [1]. Vascular calcification is one of the major problems among CKD patients and contributes to the increased risk of $\mathrm{CV}$ events by a variety of mechanisms including increased arterial stiffness due to medial calcification or plaque vulnerability linked with atherosclerotic calcification [2]. Risk factors for atherosclerosis and coronary artery disease, including age, sex, dyslipidemia, diabetes, obesity, smoking habit, high blood pressure, family history of early onset CV disease, inflammation and renal function are used to predict absolute risk for $\mathrm{CV}$ disease both in CKD patients and general population [3]. Emerging nontraditional risk factors for risk assessment include high sensitive C-reactive protein (hsCRP), fibrinogen, lipoprotein A, interleukin-6 (IL-6) and plasminogen activator inhibitor (PAI-1). The

*Corresponding author: Katarzyna Janda, Department of Nephrology, Jagiellonian University, Medical College, 31-501 Krakow, Kopernika 15c Str., Poland, Tel: +48-124247800; Fax: +48-124247941; E-mail: kasiajanda@op.pl

Received February 18, 2014; Accepted March 22, 2014; Published March 29, 2014

Citation: Janda K, Krzanowski M, Dumnicka P, Kapusta M, Klimeczek P, et al. (2014) Risk Stratification in Dialysis Patients: Coronary Artery Calcification Score Combined with High Sensitive C-Reactive Protein and Framingham Score for Cardiovascular Risk Prediction in Asymptomatic Subjects. J Clin Exp Cardiolog 5: 296. doi:10.4172/2155-9880.1000296

Copyright: $\odot 2014$ Janda K, et al. This is an open-access article distributed under the terms of the Creative Commons Attribution License, which permits unrestricted use, distribution, and reproduction in any medium, provided the original author and source are credited. 
Citation: Janda K, Krzanowski M, Dumnicka P, Kapusta M, Klimeczek P, et al. (2014) Risk Stratification in Dialysis Patients: Coronary Artery Calcification Score Combined with High Sensitive C-Reactive Protein and Framingham Score for Cardiovascular Risk Prediction in Asymptomatic Subjects. J Clin Exp Cardiolog 5: 296. doi:10.4172/2155-9880.1000296

Page 2 of 6

role of inflammation in the pathogenesis of several complications of CKD is well documented [4]. In particular, several markers have been associated with CV morbidity and vascular calcification. They include: hsCRP, IL-6, markers of oxidative stress as well as bone turnover with some evidence of a cause-effect relationship [5-7].

Coronary calcification is a marker of atherosclerosis and evaluation of CACS by Multi-Row Spiral Computed Tomography (MSCT) has been recognized as useful strategy to initiate or intensify treatment of atherosclerosis. Besides the risk of CV disease, CACS has been demonstrated to be associated with the risk of complication during $\mathrm{PCI}$, including arterial perforation, dissection and resultant stent thrombosis [2]. The non-invasive test such as CACS used for CV disease evaluation in CKD patients has the purpose of identifying Coronary Artery Disease (CAD) and evaluating the risk of CV events and death. CACS identifies calcified plaques using scattered $\mathrm{X}$-rays; it is a marker of atherosclerosis that may improve current risk assessment when added to traditional risk factors [8,9]. The aim of the study concerning Peritoneal Dialysis (PD) patients was to evaluate the association of CACS with long-term (6 years) total and CV mortality in relation to traditional CV risk factors, inflammatory markers and vascular calcification parameters.

\section{Patients and Methods}

The study group consisted of 53 asymptomatic patients ( 25 women, 28 men) with a mean age of $52 \pm 12$ years, treated with PD for a median period of 24 months (range 4 to 100 months). Patients were included according to the following criteria: age above 18 years, continuous methods of PD (CADO, CCDO), stable clinical dialysis course for at least 2 months before inclusion into the study, negative history for cardiovascular diseases and neoplasms and no active viral infection (HIV, hepatitis type B or C). PD patients were qualified during routine hospital visits and on this same day CACS was performed, as well as collection of blood samples for biochemical tests. The concentrations of albumin, fibrinogen, total cholesterol, LDL-cholesterol, HDLcholesterol, Triglycerides (TG), glucose, Osteoprotegerin (OPG), Fibroblast Growth Factor 23 (FGF23), Osteopontin (OPN), Osteocalcin (OC), intact Parathyroid Hormone (iPTH), total calcium (Ca) and Phosphates $(\mathrm{Pi})$ and peripheral blood counts were measured. Peritoneal dialysis dose was assessed using Weekly Creatinine Clearance ( $\mathrm{WCrCl})$. An echocardiography was performed in each patient within 1 month from inclusion.

Routine biochemical tests were carried out on automatic biochemical analyzers: Hitachi 917 and Modular P (Roche Diagnostics, Mannheim, Germany). Hematology parameters were measured using Sysmex XE 2100 Hematological Analyzer (Sysmex Corp. Japan). Concentrations of hsCRP were measured using the immunonephelometric method on Nephelometer BN II (Siemens Healthcare Diagnostics, Germany). Bone disease markers were determined using ELISA immunoassay. We used the following sets of reagents: OPG (Biomedica, Wien), FGF 23 (Immutopics, USA) OC (METRA, Germany), OPN (Quantikine Human Osteopontin Elisa kit, R\&D Systems). All measurements using ELISA micro-plates were performed on the Automatic Micro ELISA Reader ELX808 (BIO-TEK ${ }^{\oplus}$ Instruments, Inc., Vermont, USA). Body mass index (BMI) was calculated using the Quetelet formula. The assessment of calcifications in coronary arteries was performed by MSCT (Somaton Plus 4 Volume Zoom), using a calcium scoring program (Siemens Company, Nürnberg, Germany). The Agatston scale was employed to interpret the results, using CACS expressed in Hounsfield units (HU). Transthoracic echocardiography was performed with the Simpson's biplane method, using Vivide 7 GE Healthcare machine.

Mean Arterial Pressure (MAP) was calculated from the formula: MAP $=($ systolic blood pressure $+2 \times$ diastolic blood pressure $) / 3$ Ten-year risk of coronary artery disease (CAD) was calculated by the Framingham Risk Score (FRS) in accordance with published guidelines [10].

Data on mortality were collected during the 6 year (72 months) period. All deaths occurred in the hospital and causes of death were determined using disease history documentation.

The study was approved by the Bioethics Committee of the Jagiellonian University and all patients signed an informed consent for their participation.

\section{Statistical analysis}

Number of patients (percentage of the group) was reported for categorical variables and mean \pm standard deviation or median (lower-upper quartile) for continuous variables with normal or nonnormal distributions, as appropriate. The Shapiro-Wilk test was used to assess normality. Simple correlations were studied using Spearman coefficient. Multiple linear regression models were constructed using predictors significant in simple analysis, after log-transformation of the right-skewed variables. Survival curves were computed using the Kaplan-Meier method and compared with log-rank test. Patients who underwent renal transplantation during the study period were considered as censored observations. Unadjusted and adjusted Hazard Ratios (HR) for all-cause and cardiovascular mortality were estimated using Cox proportional regression and were reported with 95\% confidence intervals (95\% CI). HR per one unit increase was reported unless otherwise stated. Multiple Cox models were adjusted for predefined set of confounders, listed in the Results section. Nested Cox models were compared with log-likelihood statistic. Results were considered significant at $\mathrm{p} \leq 0.05$. Statistical 10 package (StatSoft, Inc., Tulsa, USA) was used for computations.

\section{Results}

Clinical characteristics of the studied group and the concentrations of selected bone markers together with the reference ranges are presented in Table 1.

CACS correlated with age of patients, FRS as well as with OPG and FGF23 levels. Other bone markers (OC, OPN) did not correlate with CACS, nor did Ca or Pi levels, Ca x Pi product and iPTH concentration (Table 2). Also, CACS did not correlate with the parameters of dialysis adequacy. In a multiple model, including age, FRS, $\log (\mathrm{OPG})$ and $\log$ (FGF23) as the independent variables, age and $\log$ (FGF23) were shown to be independent predictors of $\log$ (CACS) (beta $=0.51, \mathrm{p}=0.001$ and beta $=0.45, \mathrm{p}=0.003$, respectively). The model explained $41 \%$ of $\log$ (CACS) variance $(\mathrm{p}=0.001$ for the model).

During the six years follow-up, 16 patients (30\%) experienced nonfatal CV events, including acute myocardial infarction in 13 patients and ischemic stroke in 3 . Twenty four patients ( $45 \%$ of the group) died during the study period, including 19 patients (79\%) due to CV causes (Table 1), i.e. sudden cardiac death (7 patients), myocardial infarction (5 patients), cerebral stroke (3 patients), chronic heart failure and peripheral vascular disease ( 2 patients each). Other causes of death in these patients included: encapsulating peritoneal sclerosis (EPS) (3 patients) and infections (2 patients). The studied group included 19 patients (36\%) who were transferred to hemodialysis during the study 
Citation: Janda K, Krzanowski M, Dumnicka P, Kapusta M, Klimeczek P, et al. (2014) Risk Stratification in Dialysis Patients: Coronary Artery Calcification Score Combined with High Sensitive C-Reactive Protein and Framingham Score for Cardiovascular Risk Prediction in Asymptomatic Subjects. J Clin Exp Cardiolog 5: 296. doi:10.4172/2155-9880.1000296

\begin{tabular}{|c|c|c|c|}
\hline $\begin{array}{l}\text { Peritoneal dialysis patients } \\
(\mathrm{N}=53)\end{array}$ & Average values* & $\begin{array}{l}\text { Minimum- } \\
\text { maximum }\end{array}$ & Reference values \\
\hline Age, years & $52 \pm 12$ & $19-75$ & - \\
\hline Gender: men/women & $\begin{array}{c}28(53 \%) / 25 \\
(47 \%)\end{array}$ & & - \\
\hline $\begin{array}{l}\text { Dialysis treatment } \\
\text { duration, months }\end{array}$ & $24(15-51)$ & $4-100$ & - \\
\hline $\begin{array}{l}\text { Observation period, } \\
\text { months }\end{array}$ & $60(9-72)$ & $1-72$ & - \\
\hline All-cause mortality & $24(45 \%)$ & - & - \\
\hline Cardiovascular mortality & $19(36 \%)$ & - & - \\
\hline Active smoking & $11(21 \%)$ & - & - \\
\hline Diabetes & $12(23 \%)$ & - & - \\
\hline Hypertension & $46(87 \%)$ & - & - \\
\hline $\begin{array}{l}\text { Left ventricular ejection } \\
\text { fraction, } \%\end{array}$ & $64(60-66)$ & $45-74$ & $55-70$ \\
\hline Left ventricular mass, g & $244 \pm 78$ & $102-423$ & $\begin{array}{l}F: 67-162 \\
M: 88-224\end{array}$ \\
\hline $\begin{array}{l}\text { Left ventricular mass } \\
\text { index, } \mathrm{g} / \mathrm{m}^{2}\end{array}$ & $138 \pm 43$ & $55-246$ & $\begin{array}{l}\mathrm{F}: \text { maximum to } 110 \\
\mathrm{M}: \text { maximum to } 125\end{array}$ \\
\hline FRS, points & $8(5-10)$ & $8-16$ & - \\
\hline FRS, ten-year risk, \% & $10(6-16)$ & $1-37$ & - \\
\hline $\mathrm{BMI}, \mathrm{kg} / \mathrm{m}^{2}$ & $25.2 \pm 4.2$ & $17.2-34.2$ & $20-25$ \\
\hline $\begin{array}{l}\text { Systolic blood pressure, } \\
\mathrm{mmHg}\end{array}$ & $149 \pm 18$ & $105-180$ & $<130$ \\
\hline $\begin{array}{l}\text { Diastolic blood pressure, } \\
\mathrm{mmHg}\end{array}$ & $90 \pm 12$ & $60-115$ & $<80$ \\
\hline $\begin{array}{l}\text { Mean arterial pressure, } \\
\mathrm{mmHg}\end{array}$ & $110 \pm 11$ & $87-133$ & $75-100$ \\
\hline Hemoglobin, g/dL & $11.9 \pm 1.7$ & $7.7-15.3$ & $12-17$ \\
\hline Fibrinogen, g/L & $6.0 \pm 1.3$ & $2.9-8.6$ & $1.8-3.5$ \\
\hline WBC, $x 10^{3} / \mu \mathrm{L}$ & $7.56 \pm 2.44$ & $2.77-12.36$ & $4-10$ \\
\hline hsCRP, mg/L & $\begin{array}{l}4.18(1.29- \\
11.40)\end{array}$ & $0.16-35.9$ & $<3.17$ \\
\hline Albumin, g/L & $38 \pm 5$ & $20-47$ & $35-50$ \\
\hline Fasting glucose, $\mathrm{mmol} / \mathrm{L}$ & $4.7(4.4-5.4)$ & $3.4-15.4$ & $3.3-5.6$ \\
\hline Total cholesterol, mmol/L & $5.54 \pm 1.15$ & $3.35-9.02$ & $3.2-5.2$ \\
\hline LDL-cholesterol, mmol/L & $3.25 \pm 0.93$ & $1.45-6.35$ & $0.2-3.4$ \\
\hline HDL-cholesterol, mmol/L & $1.41 \pm 0.33$ & $0.84-2.24$ & $0.9-3.0$ \\
\hline Triglycerides, mmol/L & $1.95 \pm 0.81$ & $0.59-3.66$ & $0.2-2.3$ \\
\hline $\mathrm{Ca}, \mathrm{mmol} / \mathrm{L}$ & $2.30 \pm 0.22$ & $1.59-2.67$ & $2.1-2.4$ \\
\hline $\mathrm{Pi}, \mathrm{mmol} / \mathrm{L}$ & $1.78 \pm 0.48$ & $0.79-2.59$ & $1.1-1.8$ \\
\hline $\mathrm{Ca} \times \mathrm{Pi}, \mathrm{mmol}^{2} / \mathrm{L}^{2}$ & $4.11 \pm 1.27$ & $1.81-5.99$ & $<4.4$ \\
\hline iPTH, pg/mL & $437(198-836)$ & 3-2474 & $12-72$ \\
\hline FGF23, RU/mL & $\begin{array}{c}3013(854- \\
12015)\end{array}$ & $14.9-38798$ & no data \\
\hline OPG, pmol/L & $\begin{array}{l}9.69(8.44- \\
13.18)\end{array}$ & $3.87-27.48$ & $2.5-12.8^{* *}$ \\
\hline $\mathrm{OC}, \mathrm{ng} / \mathrm{mL}$ & $65.2(38.9-70.3)$ & $10.6-72.5$ & $\begin{array}{l}F: 3.7-10.0 \\
M: 3.4-9.1^{* *}\end{array}$ \\
\hline OPN, ng/mL & $\begin{array}{c}1475(1038- \\
1870)\end{array}$ & $559-3092$ & $49.2-175.0^{* *}$ \\
\hline CACS, Agatston units & $92(0-761)$ & $0-4555$ & $0-100$ \\
\hline WCrCl, L/week/1.73 m² & $66.7(58.8-85.7)$ & $43.2-213.8$ & $>45.0$ \\
\hline
\end{tabular}

* Number of patients (percent) is given for categorical variables; mean \pm standard deviation or median (lower-upper quartile) for continuous variables with normal or non-normal distribution, respectively.

** Reference ranges established by manufacturer.

Table 1: Characteristics of the study group at the beginning of the study and data on mortality during follow-up period.

period ( 3 of them died due to $\mathrm{CV}$ causes during the follow-up). Eleven patients $(21 \%)$ underwent successful renal transplantation and were treated as censored observations in survival analysis. Median of overall survival in the whole studied group was 72 months (lower quartile: 17 months).

Both all-cause and CV deaths were associated with higher CACS values. Especially, patients who died due to $\mathrm{CV}$ causes had much higher CACS comparing to the rest of the group: 778 (73-2476) versus $62(0-515), p=0.03$. CACS value of 800 Agatston units significantly differentiated the group into those with higher and lower risk for $\mathrm{CV}$ death $(p=0.04)$. Figure 1 shows CV survival curves of patients with CACS over 800 Agatston units versus those with lower values.

CACS significantly correlated with total and CV mortality in PD patients in simple Cox regression. The hazard ratios per 100 Agatston units' increase in CACS were as following: HR=1.03 (1.01-1.07), $\mathrm{p}=0.02$ for all-cause mortality and $\mathrm{HR}=1.05$ (1.02-1.08), $\mathrm{p}=0.003$ for CV mortality. Of the bone markers studied, only OPG significantly correlated with survival in simple analysis $[\mathrm{HR}=1.09(1.004-1.19)$, $\mathrm{p}=0.04$ for overall survival]. Also, CRP [HR=1.04 (1.01-1.07), $\mathrm{p}=0.003$ and $\mathrm{HR}=1.04$ (1.01-1.07), $\mathrm{p}=0.006$ ] and FRS [points: $\mathrm{HR}=1.13(1.001-$ 1.30), $\mathrm{p}=0.049$ and $\mathrm{HR}=1.21(1.03-1.42), \mathrm{p}=0.02$; ten-year risk: $\mathrm{HR}=1.04$ (1.001-1.08), $\mathrm{p}=0.04$ and $\mathrm{HR}=1.05$ (1.01-1.10), $\mathrm{p}=0.02]$ appeared to be significant predictors of all-cause and CV mortality in simple Cox regression. FRS was significantly higher in patients who died from $\mathrm{CV}$

\begin{tabular}{|c|c|c|}
\hline Independent variable & Spearman correlation coefficient & $\mathbf{P}$ \\
\hline Age & 0.41 & 0.002 \\
\hline Dialysis therapy duration & 0.17 & 0.2 \\
\hline FRS, points & 0.37 & 0.007 \\
\hline FRS, ten-year risk & 0.28 & 0.04 \\
\hline Ca & 0.22 & 0.1 \\
\hline Pi & 0.08 & 0.6 \\
\hline Ca x Pi & 0.12 & 0.4 \\
\hline hsCRP & 0.27 & 0.053 \\
\hline iPTH & -0.03 & 0.8 \\
\hline FGF23 & 0.43 & 0.002 \\
\hline OPG & 0.39 & 0.004 \\
\hline OC & -0.14 & 0.3 \\
\hline OPN & -0.02 & 0.9 \\
\hline
\end{tabular}

Table 2: Simple correlations of selected variables with CACS

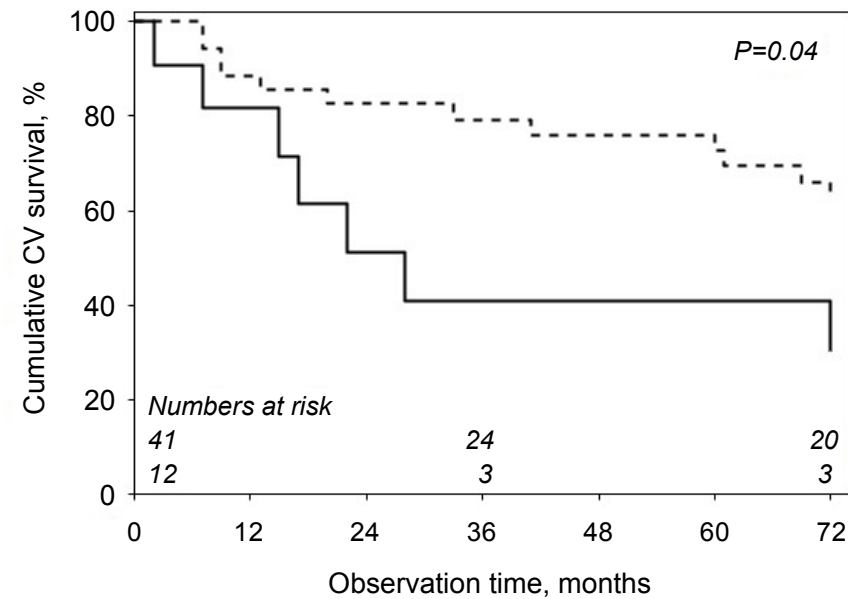

Figure 1: Kaplan-Meier survival curves for cardiovascular mortality in patients with CACS above 800 Agatston units (solid line) versus patients with lower CACS values (dashed line). Total numbers at risk at baseline, after 3 and 6 years are shown at the bottom of the graph. 
Citation: Janda K, Krzanowski M, Dumnicka P, Kapusta M, Klimeczek P, et al. (2014) Risk Stratification in Dialysis Patients: Coronary Artery Calcification Score Combined with High Sensitive C-Reactive Protein and Framingham Score for Cardiovascular Risk Prediction in Asymptomatic Subjects. J Clin Exp Cardiolog 5: 296. doi:10.4172/2155-9880.1000296

Page 4 of 6

causes [FRS, points: 10 (8-11) versus 7 (3-9); p=0.001; FRS, ten-year risk: $13(10-25)$ versus $8(4-13) ; \mathrm{p}=0.002]$.

In multiple models, CACS was shown to predict all-cause and CV mortality independently of age of patients, dialysis therapy duration, weekly creatinine clearance, $\mathrm{Ca} \times \mathrm{Pi}, \mathrm{iPTH}, \mathrm{CRP}$ and OPG concentrations as well as FRS (Table 3).

Finally, we compared three Cox models to predict CV mortality. The basic model included FRS (points) as the independent variable; the $\mathrm{R}^{2}$ for this model was 0.29 . Adding hsCRP to the model increased the $\mathrm{R}^{2}$ value to 0.45 ( $p=0.02$ in log-likelihood test). Adding CACS to the model as the third independent variable resulted in further improvement in CV mortality prediction $\left(\mathrm{R}^{2}=0.60 ; \mathrm{p}=0.02\right.$ in log-likelihood test $)$.

\section{Discussion}

Our study presents comprehensive comparison of biochemical and clinical data with calcification status (CACS) assessed by MSCT. In CKD patients, the prevalence of CAD and the incidence of major adverse $\mathrm{CV}$ events are very high. In our study both all cause and CV deaths were associated with higher CACS values. At the threshold of 800 Agatstone units, CACS identified a subgroup with a high risk of CV mortality. The importance of CACS in determining the risk of $\mathrm{CV}$ mortality is also confirmed by other researchers [9,11-14]. In the study of Budoff et al., CACS values over 400 and especially over 1000 Agatston units were associated with high risk for all-cause mortality (relative risk 5.78 and 9.36, respectively) [15]. The study group consisted of 25,253 asymptomatic individuals referred by their primary physician for Coronary Artery Calcium (CAC) scanning in order to assess CV risk. Similarly, the 1-year incidence of hard CV events (defined as myocardial infarction or coronary death) demonstrated by Wayhs et al. in patients with CACS above 1000 was $25 \%$ [16]. Lee et al. demonstrated high cardiac events rates in CKD patients with both coronary artery stenosis and high CACS at the start of dialysis [17]. In the other study, Roe et al. evaluated 112 asymptomatic renal transplant recipients with no prior history of coronary artery revascularization or myocardial infarction and assessed coronary calcifications early post-transplant and 18 months later [18]. Higher baseline CACS was associated with $\mathrm{CV}$ events and mortality, in particular patients with CACS below 100 Agatston units had better survival rates. A quarter (25.9\%) of recipients had CAC progression. Coronary calcification progression also predicted $\mathrm{CV}$ events and mortality after adjustment for diabetes, age, dialysis vintage and presence of CAC at the time of transplantation. The St. Francis Heart Study included over 4,900 patients who were followed for 4.3 years [19]. CACS above 384 Agatston units was associated with a 30 -fold increased risk for myocardial infarction or CV death, independently of FRS and hsCRP. Also, in the Prospective Army Coronary Calcium Project, in which 2000 younger participants were evaluated with electron beam tomography and followed prospectively for a mean period of 3 years, CACS was associated with a 12 -fold increased risk for hard CV events even after controlling for the FRS [20].
Consistently, in our study, participants with high CACS had higher $\mathrm{CV}$ risk and the association was independent of the age of patients, dialysis duration, weekly creatinine clearance, CRP, Ca x Pi product, iPTH and OPG concentration as well as FRS. Moreover, the addition of CACS to FRS and hsCRP significantly improved CV risk assessment in our group of $\mathrm{PD}$ patients. In addition, our study revealed a significant association of baseline hsCRP and FRS with total and CV mortality in dialysis patients. Association of inflammation with mortality in dialysis patients is well known [21-23]. The mechanisms responsible for vascular calcification include inflammation as well as bone and mineral disturbances. We did not find a correlation between CACS and hsCRP (borderline significant), as well as markers of bone turnover, except for FGF23 and OPG. Only FGF23 was shown to be an independent predictor of CACS in a multiple model. Our results are in accordance with the study of Morena et al., demonstrating the relationship between FGF23, OPG and CAC in CKD patients [24]. The univariate analysis showed that high OPG was significantly associated with moderate CAC. Severe CAC was only associated with high phosphate fractional excretion and high FGF23. In another study, Khan et al. enrolled 99 CKD patients initiating hemodialysis in which MSCT was measured at baseline and in 67 study participants after 1 year of hemodialysis treatment. FGF23 was not associated with baseline CAC, but significantly correlated with CAC progression [25]. This association persisted after adjustment for hsCRP, 25-OH vitamin D levels and the use of phosphorus binders. In the study of Masai et al., serum FGF23 levels were associated with coronary calcification independently of classical risk factors, adipocytokines and inflammatory markers in patients without CKD and diabetes mellitus [26]. Among calcium/phosphate metabolism markers, as in our study, FGF23 showed significant correlation with Agatston score and in multivariate linear regression analysis, only age and FGF23 were independently associated with the Agatston score. Authors postulated that FGF23 may also have a direct effect on progression of CAC. On the other hand, several studies reported that FGF23 is independently associated with aortic calcification in HD patients $[27,28]$. Therefore, extraordinary accumulation of FGF23 in serum may enable inhibition of the calcification process at vessel walls. Osteoblastic transformation and production of several bone proteins were demonstrated in the intima and media of calcified blood vessels obtained from dialysis patients [29]. Another hypothesis is that FGF23, which is also a bone-associated protein, may be a marker of the volume of the tissue producing it (i.e. the proliferating vascular osteoblasts and osteocytes). This may provide an explanation for the repeatedly demonstrated reproducible correlation of 'single' FGF23 measurements with the severity of vascular calcification as well as atherosclerosis [30,31]. Similar results were obtained in our study.

In our study OPG was associated with CAC score only in simple analysis. However, of bone turnover markers, only the OPG significantly correlated with mortality in simple analysis. OPG is known to regulate bone mineral metabolism but it is also associated with inflammation, CV disease and mortality. In the study of Koo et al., OPG levels were positively correlated with inflammatory markers and

\begin{tabular}{|c|c|c|c|}
\hline \multirow{2}{*}{ Independent variable } & \multicolumn{2}{|c|}{ All-cause mortality } & \multicolumn{2}{c|}{ Cardiovascular mortality } \\
\cline { 2 - 4 } & $\mathrm{HR}(95 \% \mathrm{Cl})$ & $\mathrm{PR}(95 \% \mathrm{Cl})$ & $1.05(1.01-1.10)$ \\
\hline CACS $^{*}, 100$ Agatston units & $1.04(1.01-1.08)$ & 0.02 & 0.01 \\
\hline hsCRP, $\mathrm{mg} / \mathrm{L}$ & $1.05(1.02-1.09)$ & 0.002 & $1.05(1.01-1.09)$ \\
\hline FRS, points & $1.23(1.05-1.44)$ & 0.01 & $1.33(1.10-1.61)$ \\
\hline
\end{tabular}

'In case of CACS, HR per 100 Agatston units' increase is given.

Table 3: Significant predictors of all-cause and cardiovascular mortality in multiple Cox regression. The models additionally included age of patients, dialysis therapy duration, weekly creatinine clearance, $\mathrm{Ca} \times \mathrm{Pi}$, iPTH and OPG as independent variables. 
Citation: Janda K, Krzanowski M, Dumnicka P, Kapusta M, Klimeczek P, et al. (2014) Risk Stratification in Dialysis Patients: Coronary Artery Calcification Score Combined with High Sensitive C-Reactive Protein and Framingham Score for Cardiovascular Risk Prediction in Asymptomatic Subjects. J Clin Exp Cardiolog 5: 296. doi:10.4172/2155-9880.1000296

negatively correlated with nutritional status [32]. CV events occurred in 51 of the 176 patients on peritoneal dialysis recruited to the study during a 5 year observation period. Newly developed CV events were significantly more common in patients with higher OPG levels. Cox regression analysis revealed that higher OPG level was a significant risk factor for $\mathrm{CV}$ events even after adjustments for demographic and biochemical parameters. Increased serum concentration of OPG can serve as a surrogate marker of progression of atherosclerosis and severity of vascular calcification in CKD patients [33-36]. Our results are in accordance with the study of Kurnatowska et al. demonstrating the relationship between serum OPG and CAC, as well as common carotid artery intima-media (CCA-IMT) [37]. At baseline, $70 \%$ of the $\mathrm{HD}$ patients presented detectable CAC. The patients without calcification at baseline remained calcification free at 30 months and presented lower serum OPG and FGF23 than those with CAC. A $64.4 \%$ progression of CAC was observed in all patients with $\mathrm{CAC}$ at baseline. Additionally, both increased CAC as well as CCA-IMT correlated positively with baseline and follow-up serum OPG. The patients who died had significantly higher baseline CAC and serum OPG. Increased levels of OPG can represent a response to a mineral and bone disorders in CKD patients, it may constitute a compensatory mechanism or perform protective functions $[38,39]$.

Summarizing, although limited by the low number of participants, our study provides further evidence that CACS examination may improve CV risk assessment in CKD patients in addition to hsCRP and traditional CV risk factors represented by FRS. Better identification of high-risk patients exerts the possibility of early prevention of these diseases and may lead to reduced mortality from CV disease in the population of dialysis patients. FGF23, a regulator of phosphorus metabolism, is strongly associated with CACS and may be a simple candidate biomarker of vascular calcification among dialysis patients.

\section{Conclusion}

Coronary calcium imaging is a non-invasive method of $\mathrm{CV}$ risk stratification that can accurately identify high-risk asymptomatic dialysis patients. The challenge now is to incorporate these tests into clinical practice in a manner that will improve clinical outcomes. Combination assessment of CACS, inflammatory markers as well as conventional CV risk factors as summarized by FRS at the start of dialysis treatment may contribute to early diagnosis, prevention and reduction of deaths from $\mathrm{CV}$ disease in this population.

\section{Acknowledgments}

The manuscript was not published elsewhere except a part of it was presented in abstract form as a poster during ERA-EDTA Congress. These Studies were funded by Grants K/ZDS/000597 and K/ZDS/001453.

\section{References}

1. Lamblin N, Bauters A, Fertin M, de Groote P, Pinet F, et al. (2011) Circulating levels of hepatocyte growth factor and left ventricular remodelling after acute myocardial infarction (from the REVE-2 study). Eur J Heart Fail 13: 1314-1322.

2. Kurabayashi M (2011) [Bone and calcium update; diagnosis and therapy of bone metabolism disease update. Calcification of atherosclerotic plaques: mechanism and clinical significance]. Clin Calcium 21: 43-50.

3. Wierzbicki AS (2012) New directions in cardiovascular risk assessment: the role of secondary risk stratification markers. Int J Clin Pract 66: 622-630.

4. Peters SA, Bakker M, den Ruijter HM, Bots ML (2012) Added value of CAC in risk stratification for cardiovascular events: a systematic review. Eur J Clin Invest 42: 110-116.

5. Rosário MA, Lima JJ, Parga JR, Avila LF, Gowdak LH, et al. (2010) [Coronary calcium score as predictor of stenosis and events in pretransplant renal chronic failure]. Arq Bras Cardiol 94: 236-243.
6. Stenvinkel P, Heimbürger O, Lindholm B, Kaysen GA, Bergström J (2000) Are there two types of malnutrition in chronic renal failure? Evidence for relationships between malnutrition, inflammation and atherosclerosis (MIA syndrome). Nephrol Dial Transplant 15: 953-960.

7. Sage AP, Tintut Y, Demer LL (2010) Regulatory mechanisms in vascular calcification. Nat Rev Cardiol 7: 528-536.

8. Stern L (2012) Fibroblast growth factor 23, cardiovascular disease, and inflammation. Clin J Am Soc Nephrol 7: 1061-1062.

9. Janda K, Krzanowski M1, Gajda M2, Dumnicka P3, Fedak D4, et al. (2013) Impaired fasting glucose and diabetes as predictors for radial artery calcification in end stage renal disease patients. Int J Endocrinol 2013: 969038.

10. Wilson PW, D‘Agostino RB, Levy D, Belanger AM, Silbershatz H, et al. (1998) Prediction of coronary heart disease using risk factor categories. Circulation 97: 1837-1847.

11. Greenland P, LaBree L, Azen SP, Doherty TM, Detrano RC (2004) Coronary artery calcium score combined with Framingham score for risk prediction in asymptomatic individuals. JAMA 291: 210-215.

12. Rozanski A, Gransar H, Shaw LJ, Kim J, Miranda-Peats L, et al. (2011) Impact of coronary artery calcium scanning on coronary risk factors and downstream testing the EISNER (Early Identification of Subclinical Atherosclerosis by Noninvasive Imaging Research) prospective randomized trial. J Am Coll Cardiol 57: 1622-1632.

13. Nitta K, Akiba T, Suzuki K, Uchida K, Ogawa T, et al. (2004) Assessment of coronary artery calcification in hemodialysis patients using multi-detector spiral CT scan. Hypertens Res 27: 527-533.

14. Lee HJ, Kim YJ, Hur J, Lee JW, Hong YJ, et al. (2014) Prevalence and extent of atherosclerotic coronary artery disease and related outcome based on coronary computed tomographic angiography in asymptomatic elderly patients: retrospective cohort study. Int J Cardiovasc Imaging 30: 669-676.

15. Budoff MJ, Shaw LJ, Liu ST, Weinstein SR, Mosler TP, et al. (2007) Long-term prognosis associated with coronary calcification: observations from a registry of 25,253 patients. J Am Coll Cardiol 49: 1860-1870.

16. Wayhs R, Zelinger A, Raggi $P$ (2002) High coronary artery calcium scores pose an extremely elevated risk for hard events. J Am Coll Cardiol 39: 225-230.

17. Lee JE, Lee YK, Choi EJ, Nam JS, Choi BW (2013) Usefulness of multidetector row computed tomography for predicting cardiac events in asymptomatic chronic kidney disease patients at the initiation of renal replacement therapy. ScientificWorldJournal 2: 916354

18. Roe P, Wolfe M, Joffe M, Rosas SE (2010) Inflammation, coronary artery calcification and cardiovascular events in incident renal transplant recipients. Atherosclerosis 212: 589-594

19. Arad Y, Goodman KJ, Roth M, Newstein D, Guerci AD (2005) Coronary calcification, coronary disease risk factors, C-reactive protein, and atherosclerotic cardiovascular disease events: the St. Francis Heart Study. J Am Coll Cardiol 46: 158-165.

20. do Sameiro-Faria M, Ribeiro S, Costa E, Mendonça D, Teixeira L, et al. (2013) Risk factors for mortality in hemodialysis patients: two-year follow-up study. Dis Markers 35: 791-798.

21. deFilippi C, Wasserman S, Rosanio S, Tiblier E, Sperger H, et al. (2003) Cardiac troponin $\mathrm{T}$ and $\mathrm{C}$-reactive protein for predicting prognosis, coronary atherosclerosis, and cardiomyopathy in patients undergoing long-term hemodialysis. JAMA 290: 353-359.

22. Ignjatovia AM, Cvetkovia TP, Pavlovia RM, Miloaevia ZG, et al. (2013) Endothelial dysfunction, inflammation and malnutrition markers as predictors of mortality in dialysis patients: multimarker approach. Int Urol Nephrol 45: 17151724

23. Taylor AJ, Bindeman J, Feuerstein I, Cao F, Brazaitis M, et al. (2005) Coronary calcium independently predicts incident premature coronary heart disease over measured cardiovascular risk factors: mean three-year outcomes in the Prospective Army Coronary Calcium (PACC) project. J Am Coll Cardiol 46: 807-814.

24. Morena M, Jaussent I, Halkovich A, Dupuy AM, Bargnoux AS, et al. (2012) Bone biomarkers help grading severity of coronary calcifications in non dialysis chronic kidney disease patients. PLoS One 7: e36175.

25. Khan AM, Chirinos JA, Litt H, Yang W, Rosas SE (2012) FGF-23 and the 
Citation: Janda K, Krzanowski M, Dumnicka P, Kapusta M, Klimeczek P, et al. (2014) Risk Stratification in Dialysis Patients: Coronary Artery Calcification Score Combined with High Sensitive C-Reactive Protein and Framingham Score for Cardiovascular Risk Prediction in Asymptomatic Subjects. J Clin Exp Cardiolog 5: 296. doi:10.4172/2155-9880.1000296

progression of coronary arterial calcification in patients new to dialysis. Clin $\mathrm{J}$ Am Soc Nephrol 7: 2017-2022.

26. Masai H, Joki N, Sugi K, Moroi M (2013) A preliminary study of the potential role of FGF-23 in coronary calcification in patients with suspected coronary artery disease. Atherosclerosis 226: 228-233.

27. Nasrallah MM, El-Shehaby AR, Osman NA, Fayad T, Nassef A, et al. (2013) The Association between Fibroblast Growth Factor-23 and Vascular Calcification Is Mitigated by Inflammation Markers. Nephron Extra 3: 106-112.

28. Tamei N, Ogawa T, Ishida H, Ando Y, Nitta K (2011) Serum fibroblast growth factor-23 levels and progression of aortic arch calcification in non-diabetic patients on chronic hemodialysis. J Atheroscler Thromb 18: 217-223.

29. Moe SM, Chen NX (2004) Pathophysiology of vascular calcification in chronic kidney disease. Circ Res 95: 560-567.

30. Jean G, Bresson E, Terrat JC, Vanel T, Hurot JM, et al. (2009) Peripheral vascular calcification in long-haemodialysis patients: associated factors and survival consequences. Nephrol Dial Transplant 24: 948-955.

31. Mirza MA, Hansen T, Johansson L, Ahlström H, Larsson A, et al. (2009) Relationship between circulating FGF23 and total body atherosclerosis in the community. Nephrol Dial Transplant 24: 3125-3131.

32. Koo HM, Do HM, Kim EJ, Lee MJ, Shin DH, et al. (2011) Elevated osteoprotegerin is associated with inflammation, malnutrition and new onse cardiovascular events in peritoneal dialysis patients. Atherosclerosis 219: 925930.
33. Shiota J, Izumi N, Kasahara H, Tagawa H, Chiba T, et al. (2010) Relationship between coronary and abdominal calcification score, serum osteoprotegerin (OPG), and serum tartrate-resistant acid phosphate (TRACP)-5b in pre-dialysis patients. Nihon Jinzo Gakkai Shi 52:1022-1028.

34. Janda K, Krzanowski M, Chowaniec E, KuÅsnierz-Cabala B, Dumnicka P, et al. (2013) Osteoprotegerin as a marker of cardiovascular risk in patients on peritoneal dialysis. Pol Arch Med Wewn 123: 149-155.

35. Ogawa T, Ishida H, Akamatsu M, Matsuda N, Fujiu A, et al. (2010) Progression of aortic arch calcification and all-cause and cardiovascular mortality in chronic hemodialysis patients. Int Urol Nephrol 42: 187-194.

36. Mesquita M, Demulder A, Wolff F, Mélot C, Damry N, et al. (2010) Osteoprotegerin and progression of coronary and aortic calcifications in chronic kidney disease. Transplant Proc 42: 3444-3449.

37. Kurnatowska I, Grzelak P, Kaczmarska M, StefaÅ„czyk L, Nowicki M (2011) Serum osteoprotegerin is a predictor of progression of atherosclerosis and coronary calcification in hemodialysis patients. Nephron Clin Pract 117: c297304.

38. Bennett BJ, Scatena M, Kirk EA, Rattazzi M, Varon RM, et al. (2006) Osteoprotegerin inactivation accelerates advanced atherosclerotic lesion progression and calcification in older ApoE-/- mice. Arterioscler Thromb Vasc Biol 26: 2117-2124.

39. Price PA, June HH, Buckley JR, Williamson MK (2001) Osteoprotegerin inhibits artery calcification induced by warfarin and by vitamin $\mathrm{D}$. Arterioscler Thromb Vasc Biol 21: 1610-1616. 